\title{
Baryogenesis via leptogenesis from asymmetric dark matter and radiatively generated neutrino mass
}

\author{
Nimmala Narendra, ${ }^{1, *}$ Sudhanwa Patra, ${ }^{2, \dagger}$ Narendra Sahu, ${ }^{1, \$}$ and Sujay Shil ${ }^{3,4, \S}$ \\ ${ }^{1}$ Indian Institute of Technology Hyderabad, Kandi, Sangareddy, 502285, Telangana, India \\ ${ }^{2}$ Indian Institute of Technology Bhilai, Raipur, Chhatishgarh, India \\ ${ }^{3}$ Institute of Physics, Sachivalaya Marg, Bhubaneswar, Odisha 751005, India \\ ${ }^{4}$ Homi Bhabha National Institute, Training School Complex, Anushakti Nagar, Mumbai 400085, India
}

(Received 26 June 2018; revised manuscript received 21 October 2018; published 21 November 2018)

\begin{abstract}
We propose an extension of the standard model (SM) by including a dark sector comprised of three generations of heavy right-handed neutrinos, a singlet scalar, and a singlet Dirac fermion, where the latter two particles are stable and are viable candidates of dark matter (DM). In the early Universe, the $C P$ violating out-of-equilibrium decay of heavy right-handed neutrinos to a singlet Dirac fermion and scalar in the dark sector generates a net DM asymmetry. The latter is then transported to the visible sector via a dimension-eight operator which conserves $B-L$ symmetry and is in thermal equilibrium above the sphaleron decoupling temperature. An additional light singlet scalar is introduced which mixes with the SM Higgs and paves a path for annihilating the symmetric components of the DM candidates. We discuss the constraints on singlet-doublet Higgs mixing from invisible Higgs decay, signal strength at the LHC, and the direct search of DM at terrestrial laboratories. At tree level, the neutrinos are shown to be massless since the symmetry of the dark sector forbids the interaction of right-handed neutrinos with SM particles. However, at the one-loop level, the neutrinos acquire sub-eV masses as required by the oscillation experiments.
\end{abstract}

DOI: 10.1103/PhysRevD.98.095016

\section{INTRODUCTION}

The evidence from the galaxy rotation curve, gravitational lensing, and large scale structure of the Universe irrefutably proves the existence of dark matter (DM) in a large scale ( $\gtrsim$ a few kpc) [1]. However, the microscopic picture of DM is hitherto not known. The only piece of information that we know about the DM is its relic abundance which is precisely measured by the satellite borne experiments WMAP [2] and PLANCK [3] to be $\Omega_{\mathrm{DM}} h^{2}=0.1199 \pm 0.0027$. However, a little is known about the underlying mechanism of generating the relic abundance of DM. The most considered scenario is that DM is a weakly interacting massive particle (WIMP) [4]. The latter gets thermalized in the early Universe due to its weak interaction property. As the temperature falls below its mass scale, the DM gets decoupled from the thermal bath and its density in a comoving volume remains constant

\footnotetext{
ph14resch01002@iith.ac.in

sudha.astro@gmail.com

*nsahu@iith.ac.in

§sujay@iopb.res.in
}

Published by the American Physical Society under the terms of the Creative Commons Attribution 4.0 International license. Further distribution of this work must maintain attribution to the author(s) and the published article's title, journal citation, and DOI. Funded by SCOAP . and is what we measure today. This is usually referred to as the WIMP miracle.

A curious observation about DM is that its relic density is about 5 times larger than the baryon density of the present Universe, i.e., $\Omega_{\mathrm{DM}} \approx 5 \Omega_{B}$. This implies that the relic density of DM can be generated in a similar way that the baryon asymmetry of the Universe has been generated. See, e.g., [5-9]. The observed baryon asymmetry, usually reported in terms of the baryon to photon ratio, $\eta=n_{B} / n_{\gamma}$, is given as [10],

$5.8 \times 10^{-10} \leq \eta \leq 6.6 \times 10^{-10} \quad(B B N) \quad(95 \% C L)$,

where $\eta=7.04 Y_{B}$ with $Y_{B} \equiv n_{B} / s$. Similarly, the observed DM abundance can be expressed as

$$
Y_{\mathrm{DM}} \equiv \frac{n_{\mathrm{DM}}}{s}=4 \times 10^{-10}\left(\frac{1 \mathrm{GeV}}{M_{\mathrm{DM}}}\right)\left(\frac{\Omega_{\mathrm{DM}} h^{2}}{0.11}\right) .
$$

This implies that $Y_{\mathrm{DM}} / Y_{B} \approx \mathcal{O}(1)$ if $M_{\mathrm{DM}} \sim 5 \mathrm{GeV}$. However, it can vary from a $\mathrm{GeV}$ to $\mathrm{TeV}$ depending on the magnitude of $C P$ violation in the visible and dark sectors. See, for instance, [8].

The standard model (SM), which is based on the gauge group $S U(3)_{C} \times S U(2)_{L} \times U(1)_{Y}$, is a successful theory of fundamental particles and their interactions. However, it does not explain either the DM abundance or baryon 


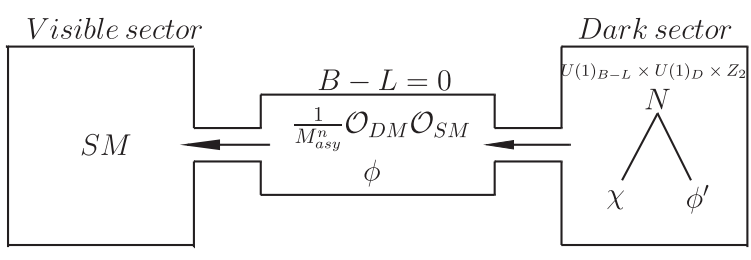

FIG. 1. Pictorial presentation of a dark sector being in thermal contact with the visible sector via Higgs portal coupling as well as higher-dimension operators, which conserve $B-L$ symmetry and are in thermal equilibrium above sphaleron decoupling temperature.

asymmetry of the Universe. Moreover, it cannot explain the nonzero masses of active neutrinos. In this paper, we make an attempt to solve these problems simultaneously in a beyond SM framework. We extend the SM by including a dark sector, as shown in Fig. 1, comprised of three generations of heavy right-handed neutrinos, a singlet scalar $\phi^{\prime}$, and a singlet Dirac fermion $\chi$. These particles are charged under an additional symmetry, $U(1)_{B-L} \times U(1)_{D} \times Z_{2}$, while remaining inert with respect to the SM gauge group. The $U(1)_{B-L}$ is a gauge symmetry, which is broken spontaneously by the vacuum expectation value of an additional scalar $\phi_{B-L}$ at a high scale, say around $10^{10} \mathrm{GeV}$, and gives Majorana masses to righthanded neutrinos, while $U(1)_{D}$ is a global symmetry and is allowed to break softly because of the higher-dimension operators. Moreover, the $U(1)_{D}$ symmetry provides a distinction between the dark sector fermions $N_{R}$ and $\chi$, which have the same charge under $U(1)_{B-L} \times Z_{2}$ symmetry. Due to $U(1)_{D}$ symmetry, both the singlet fermion $(\chi)$ and scalar $\left(\phi^{\prime}\right)$ are stabilized and become viable candidates of dark matter.

The Majorana mass of heavy right-handed neutrinos breaks $B-L$ symmetry by two units. Therefore, the $C P$ violating out-of-equilibrium decay of heavy right-handed neutrinos to $\chi \phi^{\prime}$ in the early Universe generates a net $B-L$ asymmetry $[11,12]$. The latter is then transferred to the visible sector by a dimension-eight operator [13-15], $\mathcal{O}_{8}=\frac{1}{M_{\text {asy }}^{4}} \bar{\chi}^{2}(L H)^{2}$, which is in thermal equilibrium above the sphaleron decoupling temperature. Note that the operator $\mathcal{O}_{8}$ breaks $U(1)_{D}$ symmetry softly, while it conserves $B-L$ symmetry. As a result, the $B-L$ asymmetry produced by the decay of right-handed neutrinos will be distributed between the dark and visible sectors. When the DM $\chi$ decouples from the thermal bath, the asymmetry in the two sectors gets segregated. Thus, we get a net $B-L$ asymmetry in the visible sector proportional to the $B-L$ asymmetry in the dark sector. The $B-L$ asymmetry in the visible sector gets transferred to a net baryon $(B)$ asymmetry via the sphaleron transitions, while the $B-L$ asymmetry in $\chi$ remains intact. The asymmetry in $\chi$ and $\phi^{\prime}$ combined gives rise the present day relic density of DM. An additional singlet scalar $\phi$ is introduced which mixes with the SM Higgs $H$ and paves a path for annihilating the symmetric components of $\chi$ and $\phi^{\prime}$. The abundance of the singlet scalar $\phi$ will not be present in the current Universe due to its decay to standard model particles through Higgs mixing.

Note that $N_{R}$ is odd under the $Z_{2}$ symmetry. As a result, it does not have a tree-level coupling with left-handed lepton doublets as in the type-I seesaw model [16]. However, the dimension-eight operator $\mathcal{O}_{\nu}=\frac{1}{\Lambda^{4}}\left(\overline{N_{R}} L H\right)^{2}$ is allowed, where $\Lambda$ is the scale of symmetry breaking. As we discuss in Sec. II C, this generates a Majorana mass of the light neutrinos at the one-loop level. Notice that the operator $\mathcal{O}_{\nu}$ also breaks the $U(1)_{D}$ symmetry softly.

The paper is organized as follows. In Sec. II, we introduce the model. Section IIC explains the neutrino masses. The generation of DM asymmetry is explained in Sec. III. The transfer of DM asymmetry to the visible sector is discussed in Sec. IV. In Sec. V, we describe the condition for annihilation of symmetric components of the DM. In Sec. VI, we demonstrate the constraints on model parameters from invisible Higgs decay, signal strength of a SMlike Higgs, the requirement of correct relic abundance of DM, and its direct detection. We conclude in Sec. VII.

\section{THE MODEL}

The model under consideration is based on the symmetry $\mathrm{SM} \times U(1)_{B-L} \times U(1)_{D} \times Z_{2}$, where $U(1)_{B-L}$ is a local gauge symmetry and is broken spontaneously at a high scale by the vacuum expectation value of a singlet scalar $\phi_{B-L}$, whereas $U(1)_{D}$ is a global symmetry and is allowed to break softly due to higher-dimension operators, as we discuss below. In addition to that, we extend the SM particle content by introducing a dark sector comprised of three generations of heavy right-handed neutrinos $N_{i R}$, $i=1,2,3$, a Dirac fermion $\chi$, and a singlet scalar $\phi^{\prime}$. An additional singlet scalar $\phi$ is also introduced, which mixes with the SM Higgs $H$. The particle content of the model, along with the quantum numbers, is given in Table I. Under the discrete symmetry $Z_{2}$, which remains unbroken, both $N_{R}$ and $\chi$ particles are odd. As a result, the lightest $Z_{2}$ odd particle $\chi$ is stable and is a viable candidate of DM. In addition to that, we assume $\left\langle\phi^{\prime}\right\rangle=0$. This implies $\phi^{\prime}$ is also stable due to $U(1)_{D}$ symmetry. As a result, the relics of $\chi$ and $\phi^{\prime}$ constitute the DM content of the present Universe.

TABLE I. Particles of the dark sector and their quantum numbers under the imposed symmetry.

\begin{tabular}{lcccccc}
\hline \hline Fields & $S U(3)_{C}$ & $S U(2)_{L}$ & $U(1)_{Y}$ & $U(1)_{B-L}$ & $U(1)_{D}$ & $Z_{2}$ \\
\hline$N_{R}$ & 1 & 1 & 0 & -1 & 1 & - \\
$\chi$ & 1 & 1 & 0 & -1 & $1 / 3$ & - \\
$\phi$ & 1 & 1 & 0 & 0 & 0 & + \\
$\phi^{\prime}$ & 1 & 1 & 0 & 0 & $2 / 3$ & + \\
$\phi_{B-L}$ & 1 & 1 & 0 & +2 & -2 & + \\
\hline \hline
\end{tabular}


The corresponding Lagrangian can be given as

$$
\begin{aligned}
\mathcal{L} \supset & \overline{N_{R j}} i \gamma^{\mu} D_{\mu} N_{R j}+\bar{\chi} i \gamma^{\mu} D_{\mu} \chi+\frac{1}{2}\left(\partial_{\mu} \phi\right)\left(\partial^{\mu} \phi\right) \\
& +\left(\partial_{\mu} \phi^{\prime}\right)^{\dagger}\left(\partial^{\mu} \phi^{\prime}\right)+\left(D_{\mu} \phi_{B-L}\right)^{\dagger}\left(D^{\mu} \phi_{B-L}\right) \\
& +M_{\chi} \bar{\chi} \chi+\lambda_{B-L} \phi_{B-L} \overline{\left(N_{R i}\right)^{c}} N_{R j}+\lambda_{\mathrm{DM}} \bar{\chi} \chi \phi \\
& +y_{i} \overline{N_{R i}} \chi \phi^{\prime}+\text { H.c. }-V\left(H, \phi, \phi^{\prime}\right),
\end{aligned}
$$

where

$$
D_{\mu}=\partial_{\mu}+i g_{B-L} Y_{B-L}\left(Z_{B-L}\right)_{\mu}
$$

and

$$
\begin{aligned}
V\left(H, \phi, \phi^{\prime}\right)= & -\mu_{H}^{2} H^{\dagger} H+\lambda_{H}\left(H^{\dagger} H\right)^{2}+\frac{1}{2} M_{\phi}^{2} \phi^{2} \\
& +\frac{1}{4} \lambda_{\phi} \phi^{4}+M_{\phi^{\prime}}^{2} \phi^{\prime \dagger} \phi^{\prime}+\lambda_{\phi^{\prime}}\left(\phi^{\prime \dagger} \phi^{\prime}\right)^{2} \\
& +\frac{1}{2} \lambda_{H \phi}\left(H^{\dagger} H\right) \phi^{2}+\mu_{\phi} \phi\left(H^{\dagger} H\right)+\mu_{\phi}^{\prime} \phi\left(\phi^{\prime \dagger} \phi^{\prime}\right) \\
& +\lambda_{H \phi^{\prime}}\left(H^{\dagger} H\right)\left({\phi^{\prime \dagger}}^{\prime} \phi^{\prime}\right)+\frac{\lambda_{\phi \phi^{\prime}}}{2} \phi^{2}\left(\phi^{\prime \dagger} \phi^{\prime}\right)
\end{aligned}
$$

In Eq. (4), we assume that $U(1)_{B-L}$ gauge symmetry is broken spontaneously by the vev of $\phi_{B-L}$ at a high scale, $\left\langle\phi_{B-L}\right\rangle=v_{B-L} \sim 10^{10} \mathrm{GeV}$ (say). Therefore, $\phi_{B-L}$ does not play any role in the low-energy electroweak phenomenology. However, the vev of $\phi_{B-L}$ gives super heavy masses to right-handed neutrinos as well as neutral gauge boson $Z_{B-L}$. The $B-L$ quantum numbers of $N_{R}$ and $\chi$ are the same and are taken to be -1 . However, they are distinguishable by their $U(1)_{D}$ quantum numbers.

Since $B-L$ charges of all the SM fermions are known, it is straightforward to see that the uplifting of global $U(1)_{B-L}$ symmetry of the SM to a gauge one brings in $B-L$ anomalies. In particular, the nontrivial one per family is given by [17]

$$
\begin{array}{r}
U(1)_{B-L}^{3}: 3\left[2 \times\left(\frac{1}{3}\right)^{3}-\left(\frac{1}{3}\right)^{3}-\left(\frac{1}{3}\right)^{3}\right] \\
+\left[2 \times(-1)^{3}-(-1)^{3}\right]=-1,
\end{array}
$$

where the number 3 in front is the color factor. This can be exactly canceled by introducing one right-handed neutrino per family as we did in this model. Thus, the model is anomaly free. Since $\chi$ is a vectorlike fermion, it does not introduce any additional anomaly though it is charged under $U(1)_{B-L}$.

As discussed above, the mass of heavy right-handed neutrinos is $M_{N} \gg M_{W}$, while the mass of $\chi$ is $M_{\chi}<M_{W}$. The neutral gauge boson corresponding to $B-L$ symmetry acquires a large mass $M_{Z_{B-L}} \gg M_{Z}$. In the following, we discuss the $\phi-H$ mixing on which the annihilation of the symmetric component of DM depends.

\section{A. Case-I}

The electroweak phase transition occurs as the SM Higgs acquires a vacuum expectation value (vev) $v=\langle H\rangle$. This induces a nonzero vev to $\phi$ due to the trilinear term $\mu_{\phi} \phi\left(H^{\dagger} H\right)$ as given in Eq. (4). We assume that $\langle\phi\rangle=u \ll v$. Then the quantum fluctuations around the minimum can be given as

$$
H=\left(\begin{array}{c}
0 \\
\frac{v+h}{\sqrt{2}}
\end{array}\right) \quad \text { and } \quad \phi=u+\tilde{\phi} .
$$

By minimizing the scalar potential (4), we get the vacuum expectation values,

$$
u=\frac{-\mu_{\phi} v^{2}}{M_{\phi}^{2}+\lambda_{H \phi} v^{2}},
$$

and

$$
v=\sqrt{\frac{\mu_{H}^{2}-\frac{1}{2} \lambda_{H \phi} u^{2}-\mu_{\phi} u}{2 \lambda_{H}}} .
$$

Notice that $\mu_{\phi} \propto u$. In the limit $u \rightarrow 0$, we recover the vev of the SM Higgs,

$$
v=\sqrt{\frac{\mu_{H}^{2}}{2 \lambda_{H}}} .
$$

The small vev $u$ does not affect our discussions in the following sections and, hence, we set it to zero from here on.

\section{B. Case-II}

Here, we relax the $\phi$ vev to zero, i.e., $\langle\phi\rangle=0$. Then the quantum fluctuation around the minimum is given by

$$
H=\left(\begin{array}{c}
0 \\
\frac{v+h}{\sqrt{2}}
\end{array}\right) \quad \text { and } \quad \phi=\tilde{\phi} .
$$

As a result, after the electroweak phase transition, the two scalars $h$ and $\tilde{\phi}$ mix with each other. The mass matrix is given by

$$
\left(\begin{array}{cc}
2 \lambda_{H} v^{2} & \frac{\mu_{\phi} v}{\sqrt{2}} \\
\frac{\mu_{\phi} v}{\sqrt{2}} & M_{\phi}^{2}+\frac{\lambda_{H \phi}}{2} v^{2}
\end{array}\right) .
$$

Diagonalizing the above mass matrix, we get the masses $M_{h_{1}}$ and $M_{h_{2}}$ corresponding to the physical Higgses $h_{1}$ and $h_{2}$ : 


$$
\begin{aligned}
& h_{1}=h \cos \gamma+\tilde{\phi} \sin \gamma \\
& h_{2}=-h \sin \gamma+\tilde{\phi} \cos \gamma .
\end{aligned}
$$

The mixing angle $\gamma$ can be quantified as

$$
\sin \gamma \approx \frac{\sqrt{2} \mu_{\phi} v}{2 \lambda_{H} v^{2}-M_{\phi}^{2}-\frac{\lambda_{H \phi} v^{2}}{2}} .
$$

We identify $h_{1}$ to be the SM-like Higgs with mass $M_{h_{1}}=125.18 \mathrm{GeV}$, while $h_{2}$ is the second Higgs whose mass is going to be determined from the relic abundance requirement. In fact, in Sec. VI, we obtain the light scalar mass, from the requirement of depletion of the symmetric component of the $\mathrm{DM}$, to be $M_{h_{2}} \approx 2 M_{\phi}^{\prime} \approx 2 M_{\chi} \approx$ $2.32826 \mathrm{GeV}$. In Fig. 2, we show the contours of $M_{h_{1}}=$ $125.18 \mathrm{GeV}$ (dashed lines), $M_{h_{2}}=2.32826 \mathrm{GeV}$ (solid lines), and $\sin \gamma=0.14,0.9$ (dot-dashed lines) in the plane of $\lambda_{H}$ versus $\mu_{\phi}$ for $\lambda_{H \phi}=0.1$ (meeting at point A), 0.01 (meeting at point $\mathrm{B}$ ). We see that the large range of mixing is allowed to explain simultaneously the masses of $h_{1}, h_{2}$. Later, we will see that the large mixing angles are strongly constrained by other phenomenological requirements. When the mixing goes to zero (i.e., $\mu_{\phi} \rightarrow 0$, which implies $\sin \gamma \rightarrow 0$ ), we recover the SM Higgs mass $M_{h_{1}}=2 \lambda_{H} v^{2}=$ $125.18 \mathrm{GeV}$ for $\lambda_{H}=0.13$. As the mixing angle increases (i.e., $\mu_{\phi} \neq 0$ ), we still satisfy the required masses of $h_{1}$ and $h_{2}$ with small $\lambda_{H}$. In what follows, we will take $\sin \gamma$ as the measure of mixing.

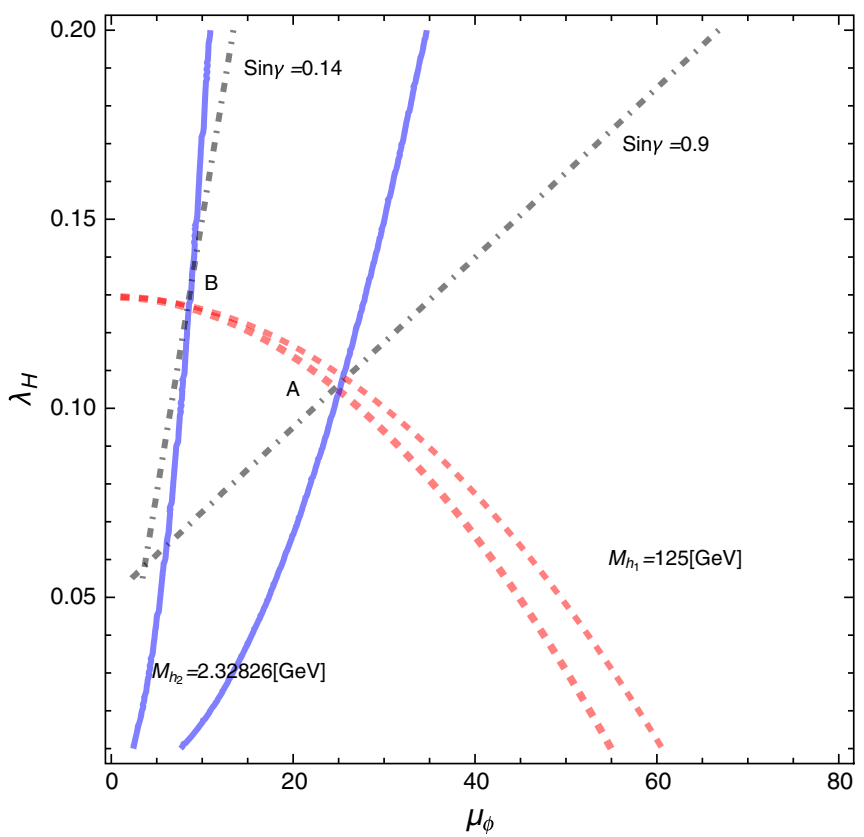

FIG. 2. Contours of $M_{h_{1}}=125.18 \mathrm{GeV}$ (dashed lines), $M_{h_{2}}=$ $2.32826 \mathrm{GeV}$ (solid lines), $\sin \gamma=0.14,0.9$ (dot dashed lines) in the plane of $\lambda_{H}$ versus $\mu_{\phi}$. We set $M_{\phi}=0$.

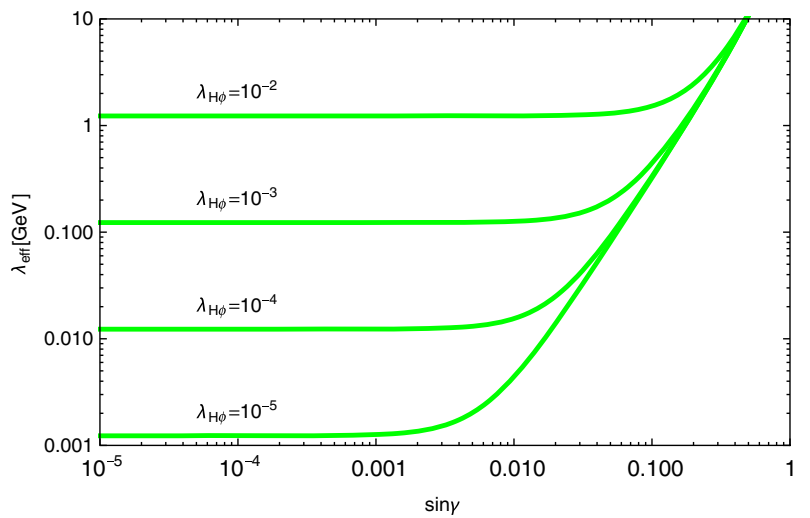

FIG. 3. Effective coupling of $h_{1}$ to $h_{2} h_{2}$ as a function of $\sin \gamma$ for $\lambda_{H}=0.13$.

The effective coupling of $h_{1} h_{2} h_{2}$ from Eq. (4) can be given as

$$
\begin{aligned}
\lambda_{\text {eff }}= & 3 \lambda_{H} v \cos \gamma \sin ^{2} \gamma+\frac{\lambda_{H \phi}}{2} v \cos ^{3} \gamma+\frac{\mu_{\phi}}{2} \sin ^{3} \gamma \\
& -\mu_{\phi} \sin \gamma \cos ^{2} \gamma-\lambda_{H \phi} v \sin ^{2} \gamma \cos \gamma .
\end{aligned}
$$

In Fig. 3, we have shown the effective coupling of SM-like Higgs to $h_{2} h_{2}$ as a function of $\sin \gamma$ for various values of $\lambda_{H \phi}$. We see that $\lambda_{\text {eff }}$ is almost independent of $\lambda_{H \phi}$ for $\sin \gamma \sim 0.1$. We will come back to this issue while calculating the invisible decay width of SM-like Higgs in Sec. VI.

\section{Neutrino masses}

The lepton number is violated by the Majorana mass term of the heavy right-handed neutrinos. Note that the term $\overline{N_{R}} \tilde{H}^{\dagger} L$ is not allowed, as $N_{R}$ is odd under the $Z_{2}$ symmetry. However, the dimension-eight operator $\mathcal{O}_{\nu}=$ $\frac{\left(\overline{N_{R}} \tilde{H}^{\dagger} L\right)^{2}}{\Lambda^{4}}$ is allowed, where $\Lambda$ is the scale of symmetry breaking. The relevant diagram generating neutrino masses radiatively is shown in Fig. $4 .{ }^{1}$

By taking $\Lambda$ as the cutoff scale, the neutrino mass can be calculated from Fig. 4 as

$$
M_{\nu}=\frac{1}{8 \pi^{2}} \frac{v^{2} M_{N}}{\Lambda^{2}}\left[1+\frac{M_{N}^{2}}{\Lambda^{2}} \log \left(\frac{M_{N}^{2}}{M_{N}^{2}+\Lambda^{2}}\right)\right],
$$

where the $v$ is the vacuum expectation value of the SM Higgs and $M_{N}$ is the mass scale of the heavy right-handed neutrino. Inverting the above formula, we get the symmetry breaking scale:

$$
\Lambda \approx 7.66 \times 10^{11} \mathrm{GeV}\left(\frac{0.1 \mathrm{eV}}{M_{\nu}}\right)\left(\frac{M_{N} / \Lambda}{0.1}\right)
$$

\footnotetext{
${ }^{1}$ See for a recent review [18].
} 


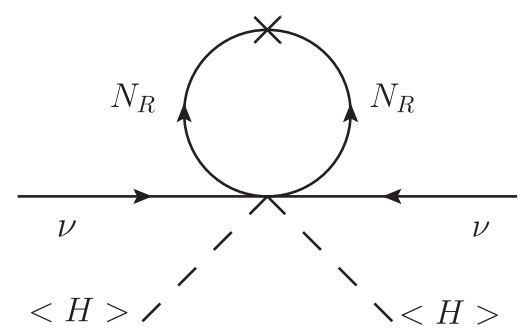

FIG. 4. Radiative neutrino mass at one-loop level, generated by the operator $\mathcal{O}_{\nu}$.

In Sec. III, we take the Majorana mass of heavy righthanded neutrinos to be $M_{N} \approx 10^{10} \mathrm{GeV}$.

\section{GENERATION OF ASYMMETRY IN DARK MATTER SECTOR}

In the early Universe, the right-handed neutrinos at a temperature above their mass scales are assumed to be in thermal equilibrium. As the Universe expands, the temperature falls. As a result, the right-handed neutrinos, below their mass scales, go out of equilibrium and decay through the process: $y_{i} \bar{N}_{R i} \chi \phi^{\prime}+$ H.c.. Without loss of generality we choose the mass basis of right-handed neutrinos to be diagonal. In this basis, the heavy Majorana neutrinos are defined by $N_{i}=\frac{1}{\sqrt{2}}\left[N_{i R}+\left(N_{i R}\right)^{c}\right]$ and hence their decay violate $B-L$ by two units. In the mass basis of $N_{1}$, the decay rate of $N_{1}$ is given by: $\Gamma_{1}=\frac{\left(y^{\dagger} y\right)_{11}}{16 \pi} M_{1}$. Comparing it with the Hubble expansion parameter $H=1.67 g_{*}^{1 / 2} T^{2} / M_{\mathrm{Pl}}$ at $T \sim M_{1}$, we get the out-of-equilibrium condition: $y \lesssim \mathcal{O}\left(10^{-3}\right) \sqrt{M_{1} / 10^{10} \mathrm{GeV}}$. Thus depending on the mass of right-handed neutrinos the decoupling epoch can be different. We assume a normal hierarchy among the heavy Majorana neutrinos. As a result, the $C P$-violating decay of lightest heavy neutrino $\left(N_{1}\right)$ to $\phi^{\prime}$ and $\chi$, generates a net asymmetry in $\chi$ and $\phi^{\prime}$. Since both $\chi$ and $\phi^{\prime}$ are stable, the asymmetry in $\chi$ and $\phi^{\prime}$ together represents the DM abundance.

The $C P$ asymmetry in the decay of $N_{1}$ arises via the interference of a tree-level diagram with one-loop self energy and vertex diagrams as shown in Fig. 5. The asymmetry $\epsilon_{\chi}$ is estimated to be [19]

$$
\begin{aligned}
\epsilon_{\chi} & =\frac{\Gamma\left(N_{1} \rightarrow \chi \phi^{\prime}\right)-\Gamma\left(N_{1} \rightarrow \bar{\chi} \phi^{\prime}\right)}{\Gamma_{N_{1}}} \\
& \simeq-\frac{1}{8 \pi}\left(\frac{M_{1}}{M_{2}}\right) \frac{\operatorname{Im}\left[\left(y^{\dagger} y\right)^{2}\right]_{12}}{\left(y^{\dagger} y\right)_{11}} .
\end{aligned}
$$

where we assume $M_{1} \ll M_{2} \ll M_{3}$, and $M_{i}, i=1,2,3$ are the masses of heavy right-handed neutrinos. Thus, below the mass scale of $N_{1}$, we get a net $B-L$ asymmetry: $[20,21]$
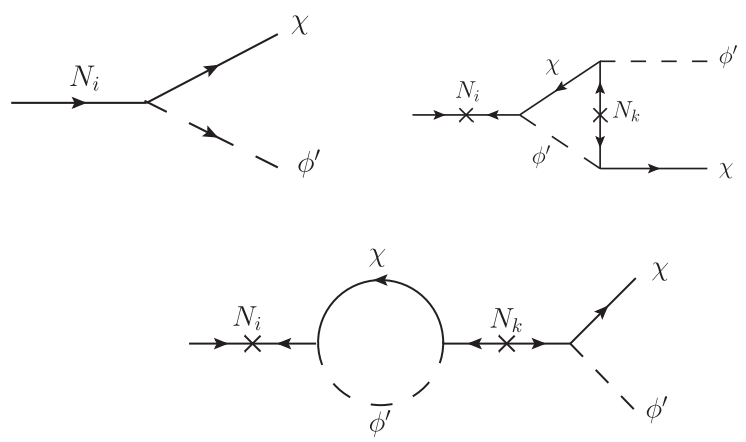

FIG. 5. CP-violation arising from the interference of tree-level diagram with vertex and self energy correction diagrams in the decay of $N_{1}$.

$$
\left(n_{B-L}\right)_{\text {total }}=\epsilon_{\chi} \kappa s \times \frac{n_{N_{1}}^{e q}(T \rightarrow \infty)}{s}
$$

where $\left(n_{N_{1}}^{e q} / s\right)(T \rightarrow \infty)=135 \zeta(3) /\left(4 \pi^{4} g_{*}\right)$ is the relativistic equilibrium abundance of $N_{1} . \kappa$ is the washout factor, arises via inverse decay and scattering processes and $s=$ $\left(2 \pi^{2} / 45\right) g_{*} T^{3}$ is the entropy density. Depending on the strength of Yukawa coupling, the value of $\kappa$ can vary between 0 to 1 . However, for definiteness we choose $\kappa=0.01$. The details of $B-L$ asymmetry generated in the dark sector can be obtained by solving the required Boltzmann equations [20], which is beyond the scope of this paper. The generated $B-L$ asymmetry will be distributed between visible and dark sectors via a higherdimension operator as we introduce in Sec. IV.

\section{ASYMMETRY TRANSFER FROM DARK SECTOR TO VISIBLE SECTOR}

The asymmetry generated via the decay of lightest heavy Majorana neutrino $N_{1}$ can be transferred to the visible sector by a higher-dimension operator [14]:

$$
\mathcal{O}_{8}=\frac{1}{M_{\text {asy }}^{4}} \bar{\chi}^{2}(L H)^{2}
$$

Depending on the value of $M_{\text {asy }}$, the transfer operator will decouple from thermal plasma at different temperatures. We can find the decoupling temperature by comparing the interaction rate of the transfer operator with the Hubble expansion rate of the universe at the decoupling epoch $T_{D}$. For the operator (18), the rate of interaction between visible and dark sector at the decoupling epoch $T_{D}$ is given as

$$
\Gamma_{\mathrm{D}} \simeq\left(\frac{T_{D}^{4}}{M_{\mathrm{asy}}^{4}}\right)^{2} T_{D}
$$

where $M_{\mathrm{Pl}}$ is the Planck mass. By comparing the above interaction rate with the Hubble expansion parameter $H=1.67 g_{*}^{1 / 2} T_{D}^{2} / M_{\mathrm{Pl}}$ we get 


$$
M_{\mathrm{asy}}^{8}>M_{\mathrm{Pl}} T_{D}^{7} .
$$

We assume that $T_{D} \gtrsim T_{\mathrm{sph}}$, where $T_{\mathrm{sph}}$ is the sphaleron decoupling temperature. For Higgs mass $M_{h_{1}}=125.18 \mathrm{GeV}$, the sphaleron decoupling temperature is $T_{\mathrm{sph}} \geq M_{W}$. As a result, from Eq. (20), we get the constraint on $M_{\text {asy }}$ to be $M_{\text {asy }}=0.9 \times 10^{4} \mathrm{GeV}=M_{\text {asy }}^{*}$ for $T_{D}=M_{W}$. In other words, Eq. (20) indicates that, if $M_{\text {asy }}>M_{\text {asy }}^{*}$, then the interaction rate of transfer operator will be in thermal equilibrium for $T>T_{D}$. The same condition also implies that the processes allowed by the operator will remain out-ofequilibrium below electroweak phase transition. Notice that the estimation of Eq. (20) holds only for the case where $\chi$ mass is much smaller than $T_{D}$. However, if one were to study heavier $\chi$, chemical decoupling can take place when the number density of $\chi$ becomes Boltzmann suppressed. See for instance [22].

The asymmetry in the equilibrium number densities of particle $n_{i}$ and antiparticle $\bar{n}_{i}$ can be given as

$n_{i}-\bar{n}_{i}=\frac{g_{i}}{2 \pi^{2}} \int_{0}^{\infty} d q q^{2}\left[\frac{1}{e^{\frac{E_{i}(q)-\mu_{i}}{T}} \pm 1}-\frac{1}{e^{\frac{E_{i}(q)+\mu_{i}}{T}} \pm 1}\right]$

where the $g_{i}$ is the internal degrees of freedom of the particle species $i$. In the above equation, $E_{i}$ and $q_{i}$ are the energy and momentum of the corresponding particle species $i$. In the approximation of a weakly interacting plasma, where $\beta \mu_{i} \ll 1, \beta \equiv 1 / T$, we get [4]

$$
\begin{aligned}
n_{i}-\bar{n}_{i} & \sim \frac{g_{i} T^{3}}{6} \times\left[2 \beta \mu_{i}+\mathcal{O}\left(\left(\beta \mu_{i}\right)^{3}\right)\right. \text { bosons } \\
& \sim \frac{g_{i} T^{3}}{6} \times\left[\beta \mu_{i}+\mathcal{O}\left(\left(\beta \mu_{i}\right)^{3}\right)\right. \text { fermions. }
\end{aligned}
$$

By comparing Eq. (22) with Eq. (17), we see that $\beta \mu \sim k \epsilon_{\chi} \ll 1$. This justifies the weak interaction of thermal plasma. We will comeback to this issue at the end of this section.

Now we will estimate the $B$ asymmetry in the visible sector at a temperature above the sphaleron decoupling temperature. To find that we will use the chemical equilibration [23] between different fermions until sphaleron decoupling temperature as discussed below. All the lefthanded charged lepton $e_{i L}, \forall i$, right-handed charged lepton $e_{i R}, \forall i$, left-handed neutrino $\nu_{i L}, \forall i$, left-handed up-type quark $u_{i L}, \forall i$, right-handed up-type quark $u_{i R}, \forall i$, left-handed down-type quark $d_{i L}, \forall i$, righthanded down-type quark $d_{i R}, \forall i, W^{ \pm}, Z$-boson, photon $(\gamma)$, Higgs $\operatorname{boson}(h)$ are in thermal equilibrium until sphaleron decoupling temperature. Here the index $i=1$, 2, 3 is written for three generations. All three generations up-type quark have the same chemical potential, all the three generations down-type quark have the same chemical potential. Similarly, all three left-handed neutrinos have the same chemical potential. But the three different charge leptons may have different chemical potential. So we omit index $i$ from chemical potential of quarks and neutrinos. The chemical potential of physical Higgs boson, $Z$ boson and photon are set to zero.

Below the electroweak phase transition, the Yukawa interactions can be given as

$$
\begin{aligned}
\mathcal{L}_{\text {Yukawa }}= & g_{e_{i}} \bar{e}_{i L} h e_{i R}+g_{u_{i}} \bar{u}_{i L} h u_{i R} \\
& +g_{d_{i}} \bar{d}_{i L} h d_{i R}+\text { H.c., }
\end{aligned}
$$

which gives the following chemical potential condition,

$$
0=\mu_{h}=\mu_{u_{L}}-\mu_{u_{R}}=\mu_{d_{L}}-\mu_{d_{R}}=\mu_{e_{i L}}-\mu_{e_{i R}} .
$$

Thus we see that for quark and charge leptons the lefthanded and right-handed fields have the same chemical potential. Sphaleron transitions are efficient down to the decoupling temperature $T_{\text {sph }}$ and, hence, we get

$$
\mu_{u_{L}}+2 \mu_{d_{L}}+\mu_{\nu}=0 .
$$

At a temperature below electroweak phase transition the electric charge neutrality of the Universe holds. However, at this epoch, the top quark is already decoupled from the thermal plasma and, hence, does not take part in the charge neutrality condition. Therefore, we get

$$
\begin{aligned}
Q & =4\left(\mu_{u_{L}}+\mu_{u_{R}}\right)+6 \mu_{W}-3\left(\mu_{d_{L}}+\mu_{d_{R}}\right)-\sum_{i=1}^{3}\left(\mu_{e_{i L}}+\mu_{e_{i R}}\right) \\
& =0 .
\end{aligned}
$$

The charge current interactions:

$$
\mathcal{L}_{\text {int }}^{(W)}=g W_{\mu}^{+} \bar{u}_{L} \gamma^{\mu} d_{L}+g W_{\mu}^{+} e_{i L} \gamma^{\mu} \bar{\nu}_{e_{i L}} .
$$

are also in thermal equilibrium below electroweak phase transition down to sphaleron decoupling temperature and hence satisfies the following chemical equilibrium condition:

$$
\begin{aligned}
& \mu_{W}=\mu_{u_{L}}-\mu_{d_{L}}, \\
& \mu_{W}=\mu_{\nu}-\mu_{e_{i L}}, \quad \forall i .
\end{aligned}
$$

Thus, Eq. (29) ensures that three generations of charge leptons also have the same chemical potential.

Thus solving the Eqs. (24)-(29), we get the total baryon and lepton number densities in the visible sector:

$$
n_{B}=-\frac{90}{29} \mu_{\nu} \quad \text { and } \quad n_{L}=\frac{201}{29} \mu_{\nu} .
$$

In Eq. (30), we have dropped the common factor $\frac{g_{i} T^{3}}{6} \times \beta$ and follow the same notation throughout the draft as we are 
interested in the ratio of densities, rather than their individual values. From the above Eq. (30), we get the total $B-L$ asymmetry in the visible sector $n_{B-L}$ :

$$
\left(n_{B-L}\right)_{\mathrm{vis}}=-\frac{291}{29} \mu_{\nu} .
$$

Moreover, from Eqs. (30) and (31), we get the total baryon asymmetry:

$$
n_{\mathrm{B}}=\frac{30}{97}\left(n_{B-L}\right)_{\mathrm{vis}}
$$

We assume that, the dark matter $\chi$ is also in thermal equilibrium with the visible sector via the dimension-eight operator $\mathcal{O}_{8}$ until the sphaleron decoupling temperature $T_{\text {sph }}>M_{W}$. This gives chemical equilibrium condition:

$$
-\mu_{\chi}+\mu_{\nu}=0
$$

Thus, from Eqs. (31) and (33), we get the number density of $\chi$ asymmetry, which is also the $B-L$ number density in the dark sector:

$$
n_{\chi}=\left(n_{B-L}\right)_{\mathrm{dark}}=-2 \mu_{\chi}=\frac{58}{291}\left(n_{B-L}\right)_{\mathrm{vis}} .
$$

The total $n_{B-L}$ of the Universe, generated by the $C P$ violating out-of-equilibrium decay of the lightest righthanded neutrino $\left(N_{1}\right)$, is the sum of $n_{B-L}$ in the visible and dark sectors. Therefore, we get

$$
\begin{aligned}
\left(n_{B-L}\right)_{\mathrm{total}} & =\left(n_{B-L}\right)_{\mathrm{vis}}+\left(n_{B-L}\right)_{\mathrm{dark}} \\
& =\left(n_{B-L}\right)_{\mathrm{vis}}+\frac{58}{291}\left(n_{B-L}\right)_{\mathrm{vis}} \\
& =\frac{349}{291}\left(n_{B-L}\right)_{\mathrm{vis}} .
\end{aligned}
$$

Comparing Eq. (35) with Eq. (17) and using Eq. (32), we get the required asymmetry for observed DM abundance $\epsilon_{\chi}=141.23(\eta / \kappa)\left(s / n_{N_{1}}^{e q}(T \rightarrow \infty)\right)$. Thus for $\kappa \sim 0.01$ we get $\epsilon_{\chi} \sim 10^{-6}$. This is in accordance with the weakly interacting plasma with $\beta \mu \sim \epsilon_{\chi} \approx 10^{-6}$. Using Eq. (35) in Eqs. (32) and (34), we can get,

$$
n_{\mathrm{B}}=\frac{90}{349}\left(n_{B-L}\right)_{\mathrm{total}}, \quad n_{\chi}=\frac{58}{349}\left(n_{B-L}\right)_{\mathrm{total}}
$$

The Asymmetry generated in $\phi^{\prime}$ can be written as,

$$
n_{\phi^{\prime}}=\left(n_{B-L}\right)_{\text {total }}
$$

The ratio of DM to baryon abundance, given by WMAP and the PLANCK data, to be $\Omega_{\mathrm{DM}} / \Omega_{\mathrm{B}} \approx 5$. This implies from Eqs. (36) and (37),

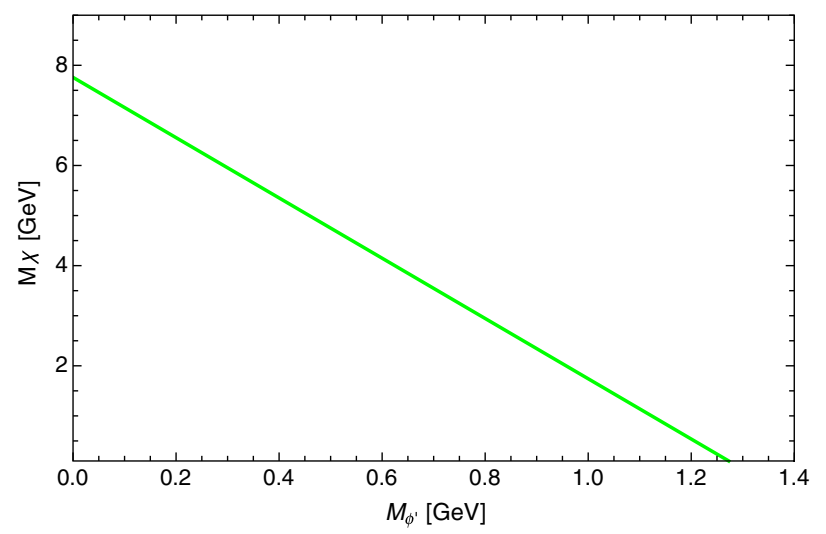

FIG. 6. Allowed mass range for both DM candidates.

$$
M_{\chi}=\frac{450 M_{p}-349 M_{\phi^{\prime}}}{58}
$$

where $M_{p}$ is the proton mass. We have shown the allowed range of masses of $\chi$ and $\phi^{\prime}$ in Fig. 6. In what follows, we take $M_{\chi}=M_{\phi^{\prime}} \approx 1 \mathrm{GeV}$.

\section{ANNIHILATION OF THE SYMMETRIC COMPONENT OF THE DARK MATTER}

The symmetric component of $\chi$ and $\phi^{\prime}$ can be efficiently depleted through the $\phi$ mediated interactions. In particular, $\phi-H$ mixing provides a portal for annihilation of $\chi$ and $\phi^{\prime}$ to the SM particles. We show that when the extra scalar mass $\left(M_{h_{2}}\right)$ is twice of the DM mass we get Breit-Wigner enhancement in the cross section which actually annihilates the symmetric component of the DM candidates, as shown in Figs. 7 and 8.

The annihilation cross section for the process $\bar{\chi} \chi \rightarrow \bar{f} f$ is given by

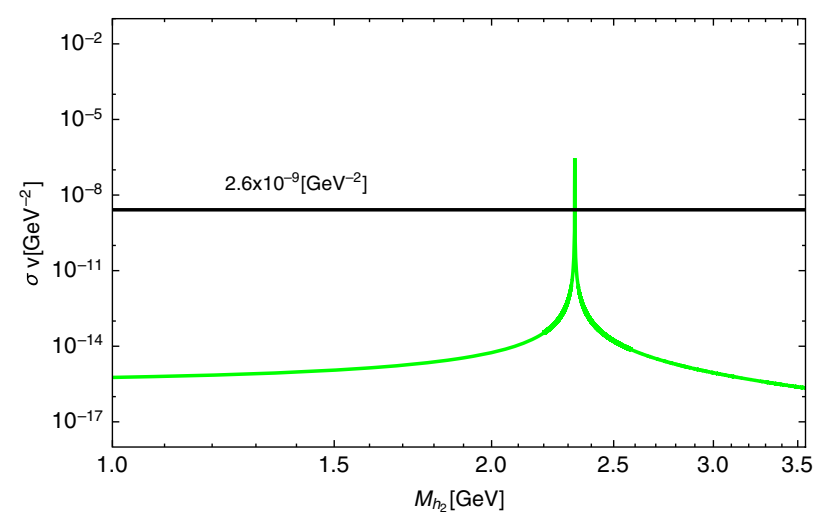

FIG. 7. The annihilation cross section of $\bar{\chi} \chi \rightarrow \bar{f} f$ as a function of $M_{h_{2}}$ for a typical value of $\lambda_{\mathrm{DM}}=1 \times 10^{-2}$, $\lambda_{\mathrm{H} \phi^{\prime}}=1 \times 10^{-3}, \mu_{\phi^{\prime}}=1 \times 10^{-3}$ and $\sin \gamma=0.1$. 


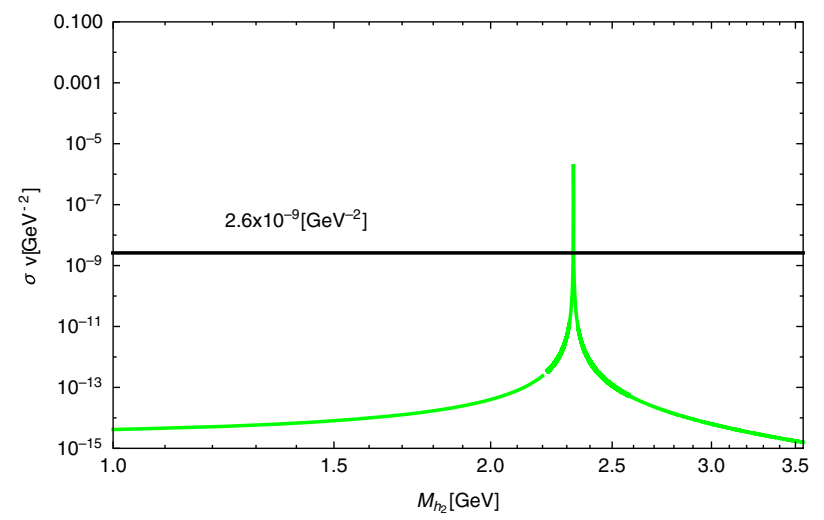

FIG. 8. The annihilation cross section of $\overline{\phi^{\dagger}} \phi^{\prime} \rightarrow \bar{f} f$ as a function of $M_{h_{2}}$ for a typical value of $\lambda_{\mathrm{DM}}=1 \times 10^{-2}$, $\lambda_{\mathrm{H} \phi^{\prime}}=1 \times 10^{-3}, \mu_{\phi^{\prime}}=1 \times 10^{-3}$ and $\sin \gamma=0.1$.

$$
\begin{aligned}
\sigma_{\chi} v= & \frac{\sqrt{s-4 M_{f}^{2}}}{16 \pi s \sqrt{s}} \\
& \times \frac{\lambda_{\mathrm{DM}}^{2} \lambda_{f}^{2} \cos ^{2} \gamma \sin ^{2} \gamma}{\left[\left(s-M_{h_{1}}^{2}\right)^{2}+\Gamma_{h_{1}}^{2} M_{h_{1}}^{2}\right]\left[\left(s-M_{h_{2}}^{2}\right)^{2}+\Gamma_{h_{2}}^{2} M_{h_{2}}^{2}\right]} \\
& \times\left\{\left[2 s-\left(M_{h_{1}}^{2}+M_{h_{2}}^{2}\right)\right]^{2}+\left[\Gamma_{h_{1}} M_{h_{1}}+\Gamma_{h_{2}} M_{h_{2}}\right]^{2}\right\} \\
& \times\left\{\left(s-2 M_{\chi}^{2}\right)\left(s-2 M_{f}^{2}\right)-2 M_{f}^{2}\left(s-2 M_{\chi}^{2}\right)\right. \\
& \left.-2 M_{\chi}^{2}\left(s-2 M_{f}^{2}\right)+4 M_{\chi}^{2} M_{f}^{2}\right\}
\end{aligned}
$$

where $M_{f}$ represents the mass of SM fermions and $\lambda_{f}=M_{f} / v$. The decay width of $h_{1}$ is given by:

$$
\Gamma_{h_{1}}=\cos ^{2} \gamma \Gamma_{h_{1}}^{\mathrm{SM}}+\sin ^{2} \gamma \Gamma_{h_{1}}^{\bar{\chi} \chi}+\Gamma_{h_{1}}^{h_{2} h_{2}}+\Gamma_{h_{1}}^{\phi^{\prime \dagger} \phi^{\prime}}
$$

where $\Gamma_{h_{1}}^{\mathrm{SM}}=4.2 \mathrm{MeV}$,

$$
\begin{aligned}
& \Gamma_{h_{1}}^{\bar{\chi} \chi}=M_{h_{1}} \frac{\lambda_{\mathrm{DM}}^{2}}{8 \pi}\left[1-\frac{4 M_{\chi}^{2}}{M_{h_{1}}^{2}}\right]^{\frac{3}{2}}, \\
& \Gamma_{h 1}^{h_{2} h_{2}}=\frac{\lambda_{\mathrm{eff}}^{2}}{32 \pi M_{h_{1}}}\left[1-\frac{4 M_{h_{2}}^{2}}{M_{h_{1}}^{2}}\right]^{\frac{1}{2}}
\end{aligned}
$$

and

$$
\Gamma_{h_{1}}^{\phi^{\prime \dagger} \phi^{\prime}}=\frac{\left(\mu_{\phi}^{\prime} \sin \gamma+\lambda_{H \phi^{\prime}} v \cos \gamma\right)^{2}}{32 \pi M_{h_{1}}}\left[1-\frac{4 M_{\phi^{\prime}}^{2}}{M_{h_{1}}^{2}}\right]^{\frac{1}{2}} .
$$

The decay width of $h_{2}$ is given by:

$$
\begin{aligned}
\Gamma_{h_{2}}= & \sum_{f} \frac{C_{f} M_{h_{2}} \sin ^{2} \gamma}{8 \pi}\left(\frac{M_{f}}{v}\right)^{2}\left[1-\frac{4 M_{f}^{2}}{M_{h_{2}}^{2}}\right]^{3 / 2} \\
& +\frac{M_{h_{2}} \lambda_{\mathrm{DM}}^{2} \cos ^{2} \gamma}{8 \pi}\left[1-\frac{4 M_{\chi}^{2}}{M_{h_{2}}^{2}}\right]^{3 / 2},
\end{aligned}
$$

where $C_{f}$ accounts the color factor of SM fermions.

The annihilation cross section for the process $\phi^{\prime \dagger} \phi^{\prime} \rightarrow$ $\bar{f} f$ is given by

$$
\begin{aligned}
\sigma_{\phi^{\prime}} v= & \frac{\left(s-4 M_{f}^{2}\right)^{\frac{3}{2}}}{8 \pi s \sqrt{s}} \\
& \times\left[\frac{\lambda_{1}^{\prime 2} \lambda_{2}^{\prime 2}}{\left(s-M_{h_{2}}\right)^{2}+\Gamma_{h_{2}}^{2} M_{h_{2}}^{2}}+\frac{\lambda_{1}^{\prime \prime 2} \lambda_{2}^{\prime \prime 2}}{\left(s-M_{h_{1}}\right)^{2}+\Gamma_{h_{1}}^{2} M_{h_{1}}^{2}}\right. \\
& +2 \lambda_{1}^{\prime} \lambda_{2}^{\prime} \lambda_{1}^{\prime \prime} \lambda_{2}^{\prime \prime} \\
& \left.\times\left(\frac{\left(s-M_{h_{2}}^{2}\right)\left(s-M_{h_{1}}^{2}\right)+\Gamma_{h_{1}} M_{h_{1}} \Gamma_{h_{2}} M_{h_{2}}}{\left[\left(s-M_{h_{2}}^{2}\right)^{2}+\Gamma_{h_{2}}^{2} M_{h_{2}}^{2}\right]\left[\left(s-M_{h_{1}}^{2}\right)^{2}+\Gamma_{h_{1}}^{2} M_{h_{1}}^{2}\right.}\right)\right]
\end{aligned}
$$

where $\quad \lambda_{1}^{\prime}=\mu_{\phi}^{\prime} \cos \gamma-\lambda_{H \phi^{\prime}} v \sin \gamma, \quad \lambda_{2}^{\prime}=-\left(M_{f} / v\right) \sin \gamma$, $\lambda_{1}^{\prime \prime}=\mu_{\phi}^{\prime} \sin \gamma+\lambda_{H \phi^{\prime}} v \cos \gamma$ and $\lambda_{2}^{\prime \prime}=\left(M_{f} / v\right) \cos \gamma$. The $\Gamma_{h_{1}}$ and $\Gamma_{h_{2}}$ are given in Eqs. (40) and (44).

In our case, $\bar{\chi} \chi$ is annihilating dominantly to a pair of muons. In Eqs. (39)and (45), the unknown parameters which dominantly contribute to the annihilation cross section are the mass of $h_{2}$, i.e., $M_{h_{2}}$, and the singletdoublet Higgs mixing, i.e., $\sin \gamma$, the coupling of $h_{2}$ with $\chi$, i.e., $\lambda_{\mathrm{DM}}$, and the coupling of $h_{2}$ with $\phi^{\prime}$, i.e., $\lambda_{H \phi^{\prime}}$. However, these parameters are strongly constrained by invisible Higgs decay [24], relic abundance of DM measured by PLANCK [3] and WMAP [2], and spinindependent direct detection cross sections at XENON100 [25], LUX [26], XENON1T [27] and CRESST-II [28] and the Higgs signal strength measured at LHC [29,30]. For a typical value of the parameters $\lambda_{\mathrm{DM}}=1 \times 10^{-2}$, $\lambda_{\mathrm{H} \phi^{\prime}}=1 \times 10^{-3}, \mu_{\phi^{\prime}}=1 \times 10^{-3}$ and $\sin \gamma=0.1$, we have plotted $\sigma v$ as a function of $M_{h_{2}}$ in Figs. 7, 8, respectively.

As shown in Figs. 7, 8, the value of the $\chi$ and $\phi^{\prime}$ annihilation cross section $\sigma v<\langle\sigma|v|\rangle_{F}=2.6 \times 10^{-9} / \mathrm{GeV}^{2}$ in most of the parameter space except at the resonance, where $\sigma v>\langle\sigma|v|\rangle_{F}$. A crucial observation here is that mass of $h_{2}$ has to be twice the DM mass in order to get a large cross section via resonance. Note that a large cross section is required to deplete the symmetric component of the DM.

\section{PHENOMENOLOGICAL CONSTRAINTS}

\section{A. Higgs signal strength}

The signal strength of SM-like Higgs in a particular channel $h_{1} \rightarrow x x$ can be measured at LHC and can be defined as 


$$
\mu_{h_{1} \rightarrow x x}=\frac{\sigma_{h_{1}}}{\sigma_{h_{1}}^{\mathrm{SM}}} \frac{\mathrm{Br}_{h_{1} \rightarrow x x}}{\mathrm{Br}_{h_{1} \rightarrow x x}^{\mathrm{SM}}}=\frac{\cos ^{4} \gamma \Gamma_{h_{1}}^{\mathrm{SM}}}{\Gamma_{h_{1}}},
$$

where $\Gamma_{h_{1}}$ is given by Eq. (40). In the absence of any new physics, $\mu=1$. However, in our case, the mixing between the two Higgses can reduce the signal strength of SM-like Higgs. Therefore, the mixing cannot be arbitrarily large and can be strongly constrained from the observation. The combined signal strength is measured to be $\mu=1.17 \pm 0.1$ [29,30]. In Figs. 9 and 10, we have shown the contours of different values of $\mu$ in the planes of $\lambda_{\mathrm{DM}}$ and $\lambda_{\mathrm{H} \phi^{\prime}}$ with $\sin \gamma$, respectively. From the Figs. 9 and 10 we see that as the mixing increases the signal strength reduces accordingly. For an optimistic low value $\mu=0.80$, the allowed mixing angle can be as large as 0.28 . Thus in the rest of the draft we restrict the range of $\sin \gamma$ up to 0.3 .

\section{B. Constraints from invisible Higgs decay}

The singlet-doublet Higgs mixing in this model allows the SM-like Higgs $h_{1}$ to decay via invisible channels:

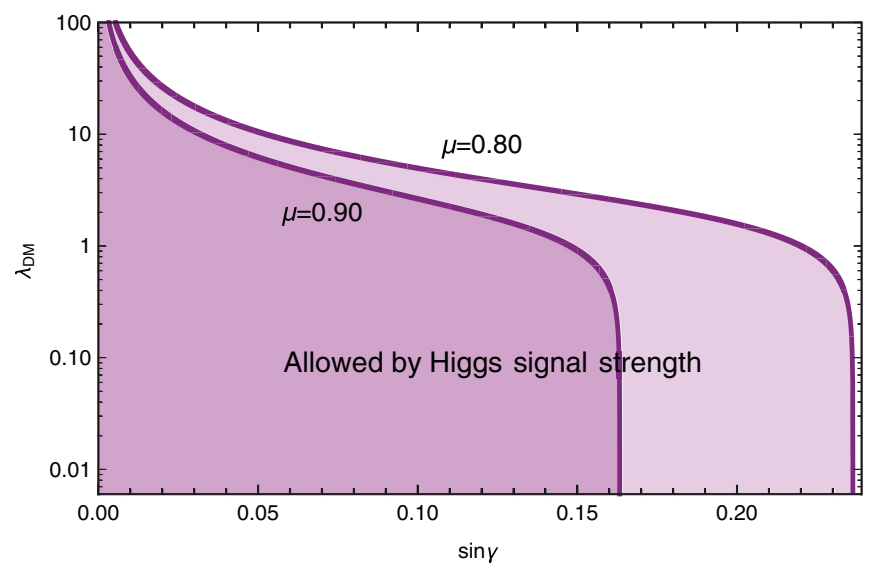

FIG. 9. Contours of signal strength $\mu$ of the SM-like Higgs $h_{1}$ in the plane of $\lambda_{\mathrm{DM}}$ versus $\sin \gamma$.

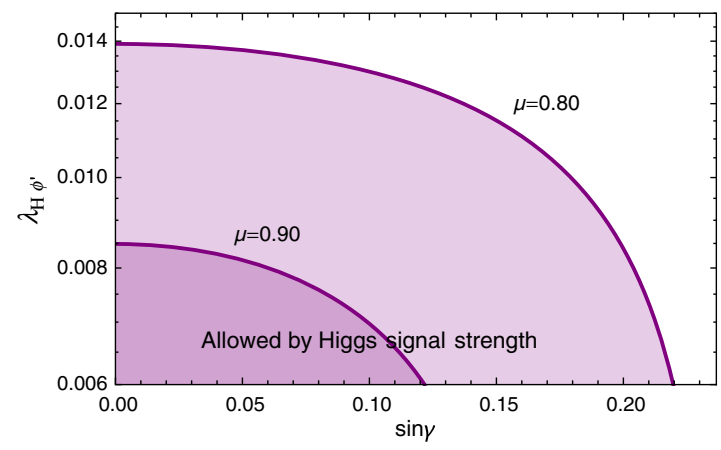

FIG. 10. Contours of signal strength $\mu$ of the SM-like Higgs $h_{1}$ in the plane of $\lambda_{\mathrm{H} \phi^{\prime}}$ versus $\sin \gamma$. $h_{1} \rightarrow h_{2} h_{2}, h_{1} \rightarrow \bar{\chi} \chi$ and $h_{1} \rightarrow \bar{\phi}^{\prime \dagger} \phi^{\prime}$. The branching ratio for the invisible Higgs decay can be defined as

$\mathrm{Br}_{\mathrm{inv}}=\frac{\sin ^{2} \gamma \Gamma_{h_{1}}^{\bar{\chi} \chi}+\left[\operatorname{Br}\left(h_{2} \rightarrow \bar{\chi} \chi+h_{2} \rightarrow \phi^{\prime \dagger} \phi^{\prime}\right)\right] \Gamma_{h_{1}}^{h_{2} h_{2}}+\Gamma_{h_{1}}^{\phi^{\prime \dagger}} \phi^{\prime}}{\cos ^{2} \gamma \Gamma_{h_{1}}^{\mathrm{SM}}+\sin ^{2} \gamma \Gamma_{h_{1}}^{\bar{\chi} \chi}+\Gamma_{h_{1}}^{h_{2} h_{2}}+\Gamma_{h_{1}}^{\phi^{\prime \dagger} \phi^{\prime}}}$,

where $\Gamma_{h_{1}}^{\bar{\chi} \chi}, \Gamma_{h_{1}}^{h_{2} h_{2}}$ and $\Gamma_{h_{1}}^{\phi^{\prime \prime} \phi^{\prime}}$ are given by Eqs. (41)-(43), respectively. Note that LHC give an upper bound to the invisible Higgs decay to be $\mathrm{Br}_{\text {inv }} \leq 24 \%$ [24]. For a given $h_{2}$ mass, the allowed invisible Higgs decay width will constraint $\lambda_{\mathrm{DM}}, \lambda_{\mathrm{H} \phi^{\prime}}$ and $\sin \gamma$ as we discuss below.

\section{Constraints from direct detection of dark matter}

The singlet-doublet scalar mixing also allows the DM $\chi$ and $\phi^{\prime}$ to scatter off the nucleus at terrestrial laboratories. The spin independent DM-nucleon scattering cross section can be written as [31-34]

$$
\sigma^{S I}=\frac{\mu_{r}^{2}}{\pi A^{2}}\left[Z f_{p}+(A-Z) f_{n}\right]^{2}
$$

Where the $\mathrm{Z}$ and $\mathrm{A}$ are the atomic and mass numbers of the target nucleus. In Eq. (48), the reduced mass $\mu_{r}=M_{\chi} m_{n} /\left(M_{\chi}+m_{n}\right)$, where $m_{n}$ is the mass of the nucleon (proton or neutron) and $f_{p}$ and $f_{n}$ are the effective interaction strengths of DM with proton and neutron of the target nucleus and are given by:

$$
f_{p, n}=\sum_{q=u, d, s} f_{T_{q}}^{p, n} \alpha_{q} \frac{m_{p, n}}{m_{q}}+\frac{2}{27} f_{T G}^{p, n} \sum_{q=c, t, b} \alpha_{q} \frac{m_{p, n}}{m_{q}},
$$

where in case of $\chi$ DM can be written as

$$
\alpha_{q}=\lambda_{\mathrm{DM}}\left(\frac{m_{q}}{v}\right)\left[\frac{1}{M_{h_{2}}^{2}}-\frac{1}{M_{h_{1}}^{2}}\right] \sin \gamma \cos \gamma
$$

where in case of $\phi^{\prime} \mathrm{DM}$ can be written as

$\alpha_{q}=\left(\frac{\mu_{\phi^{\prime}} \sin \gamma+\lambda_{H \phi^{\prime}} v \cos \gamma}{M_{h_{2}}^{2}}-\frac{\mu_{\phi^{\prime}} \cos \gamma-\lambda_{H \phi^{\prime}} v \sin \gamma}{M_{h_{1}}^{2}}\right)$

In Eq. (49) above, the $f_{T_{q}}^{p, n}$ are given by $f_{T u}^{(p)}=$ $0.020 \pm 0.004, f_{T d}^{(p)}=0.026 \pm 0.005, f_{T s}^{(p)}=0.118 \pm 0.062$, $f_{T u}^{(n)}=0.014 \pm 0.003, \quad f_{T d}^{(n)}=0.036 \pm 0.008, \quad f_{T s}^{(n)}=$ $0.118 \pm 0.062$ [35]. The coupling of DM with the gluons in target nuclei is parametrized by 


$$
f_{T G}^{p, n}=1-\sum_{q=u, d, s} f_{T_{q}}^{p, n}
$$

We summarize all the constraints from invisible Higgs decay, relic abundance of DM, and null detection of DM at CRESST-II [28] as the allowed regions in the plane of $\lambda_{\mathrm{DM}}$ versus $\sin \gamma$ in Fig. 11 and $\lambda_{\mathrm{H} \phi^{\prime}}$ versus $\sin \gamma$ in Fig. 12. We see from Fig. 11 that the region above the top purple line is not allowed by the invisible Higgs decay, where $\mathrm{Br}_{\text {inv }} \geq 24 \%$ [24]. The region below the bottom red line gives large relic abundance of DM since in this

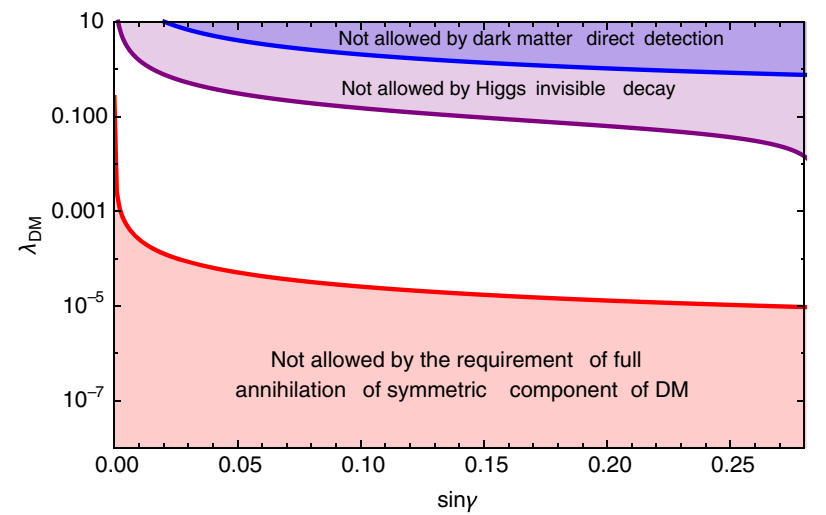

FIG. 11. Allowed regions in the plane of $\lambda_{\text {DM }}$ versus $\sin \gamma$. The region above the top purple line is disallowed by invisible Higgs decay, i.e., $\mathrm{Br}_{\mathrm{inv}} \geq 24 \%$. The region below the bottom red line is disallowed because $\sigma v<2.6 \times 10^{-9} / \mathrm{GeV}^{2}$ and give large relic abundance. The regions above the Blue line is disallowed by the spin independent direct detection cross sections at CRESST-II 2016 for DM mass $1 \mathrm{GeV}$. we fix $M_{h_{2}} \approx 2 M_{\chi}$.

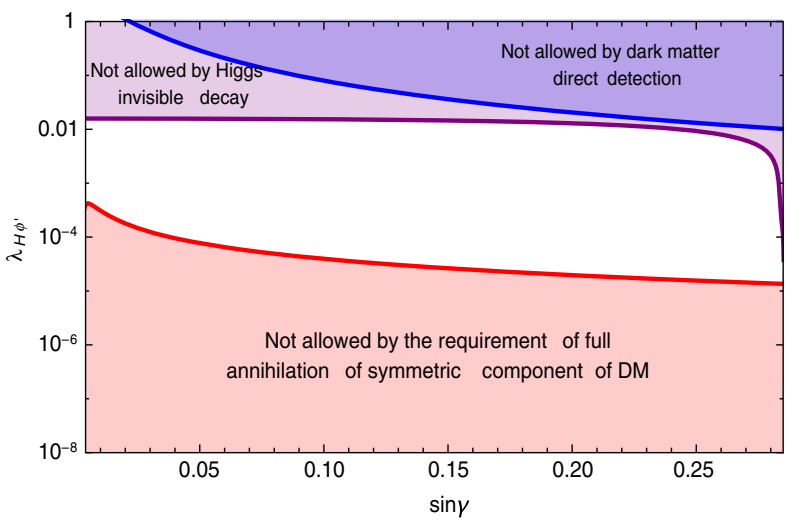

FIG. 12. Allowed regions in the plane of $\lambda_{H \phi^{\prime}}$ versus $\sin \gamma$. The region above the top purple line is disallowed by invisible Higgs decay, i.e., $\mathrm{Br}_{\text {inv }} \geq 24 \%$. The region below the bottom red line is disallowed because $\sigma v<2.6 \times 10^{-9} / \mathrm{GeV}^{2}$ and give large relic abundance. The regions above the blue line is disallowed by the spin independent direct detection cross sections at CRESST-II 2016 for DM mass $1 \mathrm{GeV}$. we fix $M_{h_{2}} \approx 2 M_{\phi}^{\prime}$. region $\sigma v\left(\bar{\chi} \chi, \phi^{\prime \dagger} \phi^{\prime} \rightarrow \bar{f} f\right)<2.6 \times 10^{-9} / \mathrm{GeV}^{2}$. Since most of the annihilation occurs at the resonance, we fix $M_{h_{2}} \approx 2 M_{\chi} \approx 2 M_{\phi}^{\prime}$. The blue line indicates the spin independent direct detection cross section from the CRESST-II [28] detector results $\sigma^{\mathrm{SI}}=10^{-38} \mathrm{~cm}^{2}$ corresponding to a DM mass: $M_{\chi}=M_{\phi^{\prime}}=1 \mathrm{GeV}$. Therefore, the region above to that line is not allowed. Thus, are we left with a white allowed patch in the plane of $\lambda_{\mathrm{DM}}$ versus $\sin \gamma$ and $\lambda_{\mathrm{H} \phi^{\prime}}$ versus $\sin \gamma$.

\section{CONCLUSION}

In this paper, we extended the standard model by including a dark sector which consists of three generations of heavy right-handed neutrinos $N_{i R}, i=1,2,3$, a singlet Dirac fermion $\chi$, and a singlet scalar $\phi^{\prime}$, where the latter two particles represent the DM. These particles are charged under an extended symmetry $U(1)_{B-L} \times U(1)_{D} \times Z_{2}$, while remaining inert with respect to the SM symmetry. An additional singlet scalar $\phi_{B-\mathrm{L}}$ was introduced to break the $U(1)_{B-L}$ gauge symmetry at a high scale, say $10^{10} \mathrm{GeV}$. The breaking of $B-L$ symmetry at a high scale not only gave large Majorana masses to heavy righthanded neutrinos but also made $Z_{B-L}$ super heavy. The global $U(1)_{D}$ symmetry, which was softly broken by dimension-eight operators, provides a distinction between $N_{R}$ and $\chi$ since they carry the same charges under the $U(1)_{B-L} \times Z_{2}$.

In the early Universe, the $C P$-violating out-ofequilibrium decay of lightest heavy right-handed neutrino to $\chi$ and $\phi^{\prime}$ generates a net DM asymmetry. The latter is then transferred to the visible sector via a dimension-eight operator $(\bar{\chi} L H)^{2} / M_{\text {asy }}^{4}$ which conserves $B-L$ symmetry and is in thermal equilibrium down to the sphaleron decoupling temperature $T_{\mathrm{sph}} . B+L$ violating sphaleron transitions are in thermal equilibrium down to a temperature $T_{\mathrm{sph}}$ and, hence, can convert the $B-L$ asymmetry in the visible sector to a net $B$ asymmetry while the $B-L$ asymmetry in the dark sector remains untouched. As a result, we get a net asymmetric DM abundance (given in terms of $B-L$ asymmetry) comparable to baryon asymmetry for $M_{\chi} \sim M_{\phi}^{\prime} \sim M_{p}$, where $M_{p}$ represents the proton mass. An additional light singlet scalar $\phi$ was introduced, which helped in annihilating the symmetric component of the DM through its mixing with the SM Higgs. We found that the efficient annihilation of the symmetric component of DM requires the singlet scalar mass to be around twice the DM mass irrespective of all other parameters in the model. Since the observed DM abundance gives the DM mass $M_{\chi}=M_{\phi^{\prime}} \approx 1 \mathrm{GeV}$, we get the singlet scalar mass $\approx 2 \mathrm{GeV}$, which can be searched at the collider and via an indirect gamma ray search.

The neutrinos are massless at the tree level since the right-handed neutrinos are odd under the $Z_{2}$ symmetry and 
are decoupled from the visible sector. However, at the oneloop level, the neutrinos acquired masses via a dimensioneight operator $\left(\bar{N}_{R} L H\right)^{2} / \Lambda^{4}$. We showed that sub-eV masses of neutrinos require the $B-L$ breaking scale to be around $\Lambda \approx 10^{11} \mathrm{GeV}$.

\section{ACKNOWLEDGMENTS}

S. S. thanks Arunansu Sil, Manimala Mitra, Kirtiman Ghosh, Pankaj Agrawal, Aruna Kumar Nayak, Debottam Das, and Bhupal Dev for useful discussions.
[1] G. Jungman, M. Kamionkowski, and K. Griest, Phys. Rep. 267, 195 (1996); G. Bertone, D. Hooper, and J. Silk, Phys. Rep. 405, 279 (2005).

[2] G. Hinshaw et al. (WMAP Collaboration), Astrophys. J. Suppl. Ser. 208, 19 (2013).

[3] P. A. R. Ade et al. (Planck Collaboration), Astron. Astrophys. 594, A13 (2016).

[4] E. W. Kolb and M. S. Turner, The Early Universe (AddisonWesley, Reading, MA, 1989).

[5] S. Nussinov, Phys. Lett. 165B, 55 (1985); K. Griest and D. Seckel, Nucl. Phys. B283, 681 (1987); B296, 1034 (1988); R. S. Chivukula and T. P. Walker, Nucl. Phys. B329, 445 (1990); S. Dodelson, B. R. Greene, and L. M. Widrow, Nucl. Phys. B372, 467 (1992); S. M. Barr, Phys. Rev. D 44, 3062 (1991); D. B. Kaplan, Phys. Rev. Lett. 68, 741 (1992); H. K. Dreiner and G. G. Ross, Nucl. Phys. B410, 188 (1993); T. Inui, T. Ichihara, Y. Mimura, and N. Sakai, Phys. Lett. B 325, 392 (1994); S. D. Thomas, Phys. Lett. B 356, 256 (1995).

[6] R. Kitano and I. Low, Phys. Rev. D 71, 023510 (2005); K. Agashe and G. Servant, J. Cosmol. Astropart. Phys. 02 (2005) 002; N. Cosme, L. L. Honorez, and M. H. G. Tytgat, Phys. Rev. D 72, 043505 (2005); G. R. Farrar and G. Zaharijas, Phys. Rev. Lett. 96, 041302 (2006); R. Kitano, H. Murayama, and M. Ratz, Phys. Lett. B 669, 145 (2008); E. Nardi, F. Sannino, and A. Strumia, J. Cosmol. Astropart. Phys. 01 (2009) 043; H. An, S. L. Chen, R. N. Mohapatra, and Y. Zhang, J. High Energy Phys. 03 (2010) 124; T. Cohen and K. M. Zurek, Phys. Rev. Lett. 104, 101301 (2010); J. Shelton and K. M. Zurek, Phys. Rev. D 82, 123512 (2010); H. Davoudiasl, D. E. Morrissey, K. Sigurdson, and S. Tulin, Phys. Rev. Lett. 105, 211304 (2010); N. Haba and S. Matsumoto, Prog. Theor. Phys. 125, 1311 (2011); M. R. Buckley and L. Randall, J. High Energy Phys. 09 (2011) 009; P. H. Gu, M. Lindner, U. Sarkar, and X. Zhang, Phys. Rev. D 83, 055008 (2011); M. Blennow, B. Dasgupta, E. Fernandez-Martinez, and N. Rius, J. High Energy Phys. 03 (2011) 014; J. McDonald, Phys. Rev. D 83, 083509 (2011); L. J. Hall, J. March-Russell, and S. M. West, arXiv:1010.0245; J. J. Heckman and S. J. Rey, J. High Energy Phys. 06 (2011) 120; M. T. Frandsen, S. Sarkar, and K. Schmidt-Hoberg, Phys. Rev. D 84, 051703 (2011); S. Tulin, H. B. Yu, and K. M. Zurek, J. Cosmol. Astropart. Phys. 05 (2012) 013.

[7] K. Kohri, A. Mazumdar, and N. Sahu, Phys. Rev. D 80, 103504 (2009); K. Kohri, A. Mazumdar, N. Sahu, and P. Stephens, Phys. Rev. D 80, 061302 (2009); K. Kohri and N. Sahu, Phys. Rev. D 88, 103001 (2013); M. Ibe,
S. Matsumoto, and T. T. Yanagida, Phys. Lett. B 708, 112 (2012); M. L. Graesser, I. M. Shoemaker, and L. Vecchi, J. High Energy Phys. 10 (2011) 110; D. Hooper, J. MarchRussell, and S. M. West, Phys. Lett. B 605, 228 (2005); H. Iminniyaz, M. Drees, and X. Chen, J. Cosmol. Astropart. Phys. 07 (2011) 003; N. Haba, S. Matsumoto, and R. Sato, Phys. Rev. D 84, 055016 (2011); Z. Kang, J. Li, T. Li, T. Liu, and J. M. Yang, Eur. Phys. J. C 76, 270 (2016); K. Blum, A. Efrati, Y. Grossman, Y. Nir, and A. Riotto, Phys. Rev. Lett. 109, 051302 (2012); M. Fujii and T. Yanagida, Phys. Lett. B 542, 80 (2002); T. Banks, S. Echols, and J. L. Jones, J. High Energy Phys. 11 (2006) 046; T. R. Dulaney, P. Fileviez Perez, and M. B. Wise, Phys. Rev. D 83, 023520 (2011); T. Cohen, D. J. Phalen, A. Pierce, and K. M. Zurek, Phys. Rev. D 82, 056001 (2010); B. Dutta and J. Kumar, Phys. Lett. B 699, 364 (2011); A. Falkowski, J. T. Ruderman, and T. Volansky, J. High Energy Phys. 05 (2011) 106; J. March-Russell and M. McCullough, J. Cosmol. Astropart. Phys. 03 (2012) 019; M. L. Graesser, I. M. Shoemaker, and L. Vecchi, arXiv:1107.2666; K. Kamada and M. Yamaguchi, Phys. Rev. D 85, 103530 (2012); D. G. E. Walker, arXiv:1202.2348; B. Feldstein and A. L. Fitzpatrick, J. Cosmol. Astropart. Phys. 09 (2010) 005; J. March-Russell, J. Unwin, and S. M. West, J. High Energy Phys. 08 (2012) 029; Y. Cai, M. A. Luty, and D. E. Kaplan, arXiv:0909.5499; H. An, S. L. Chen, R. N. Mohapatra, S. Nussinov, and Y. Zhang, Phys. Rev. D 82, 023533 (2010); C. Kouvaris and P. Tinyakov, Phys. Rev. Lett. 107, 091301 (2011); M. R. Buckley, Phys. Rev. D 84, 043510 (2011); S. Chang and L. Goodenough, Phys. Rev. D 84, 023524 (2011); S. Profumo and L. Ubaldi, J. Cosmol. Astropart. Phys. 08 (2011) 020; H. Davoudiasl, D. E. Morrissey, K. Sigurdson, and S. Tulin, Phys. Rev. D 84, 096008 (2011); I. Masina and F. Sannino, J. Cosmol. Astropart. Phys. 09 (2011) 021; T. Lin, H. B. Yu, and K. M. Zurek, Phys. Rev. D 85, 063503 (2012); M. R. Buckley and S. Profumo, Phys. Rev. Lett. 108, 011301 (2012); H. Davoudiasl and R. N. Mohapatra, New J. Phys. 14, 095011 (2012); N. Okada and O. Seto, Phys. Rev. D 86, 063525 (2012); T. Hugle, M. Platscher, and K. Schmitz, Phys. Rev. D 98, 023020 (2018).

[8] C. Arina and N. Sahu, Nucl. Phys. B854, 666 (2012); C. Arina, J. O. Gong, and N. Sahu, Nucl. Phys. B865, 430 (2012); C. Arina, R. N. Mohapatra, and N. Sahu, Phys. Lett. B 720, 130 (2013).

[9] See for a review: K. Petraki and R. R. Volkas, Int. J. Mod. Phys. A 28, 1330028 (2013); K. M. Zurek, Phys. Rep. 537, 91 (2014). 
[10] C. Patrignani et al. (Particle Data Group), Chin. Phys. C 40, 100001 (2016).

[11] A. D. Sakharov, Pis'ma Zh. Eksp. Teor. Fiz. 5, 32 (1967) [JETP Lett. 5, 24 (1967)]; Usp. Fiz. Nauk 161, 61 (1991) [Sov. Phys. Usp. 34, 392 (1991)].

[12] M. Fukugita and T. Yanagida, Phys. Lett. B 174, 45 (1986).

[13] D. E. Kaplan, M. A. Luty, and K. M. Zurek, Phys. Rev. D 79, 115016 (2009).

[14] W. Z. Feng, P. Nath, and G. Peim, Phys. Rev. D 85, 115016 (2012).

[15] M. Ibe, S. Matsumoto, and T. T. Yanagida, Phys. Lett. B 708, 112 (2012).

[16] P. Minkowski, Phys. Lett. 67B, 421 (1977); M. Gell-Mann, P. Ramond, and R. Slansky in Supergravity, edited by P. van Niewenhuizen and D. Freedman (North Holland, Amsterdam, 1979); T. Yanagida, in Workshop on Unified Theory and Baryon number in the Universe, edited by $\mathrm{O}$. Sawada and A. Sugamoto (KEK, Japan, 1979); R. N. Mohapatra and G. Senjanovic, Phys. Rev. Lett. 44, 912 (1980).

[17] C. Q. Geng and R. E. Marshak, Phys. Rev. D 39, 693 (1989); X. G. He, G. C. Joshi, and R. R. Volkas, Phys. Rev. D 41, 278 (1990); K. Kohri and N. Sahu, Phys. Rev. D 88, 103001 (2013).

[18] Y. Cai, J. Herrero-Garca, M. A. Schmidt, A. Vicente, and R. R. Volkas, Front. Phys. 5, 63 (2017).

[19] W. Buchmuller and M. Plumacher, Phys. Rep. 320, 329 (1999).

[20] W. Buchmuller, P. Di Bari, and M. Plumacher, Ann. Phys. (Amsterdam) 315, 305 (2005).
[21] G. F. Giudice, A. Notari, M. Raidal, A. Riotto, and A. Strumia, Nucl. Phys. B685, 89 (2004).

[22] N. Bernal, C.S. Fong, and N. Fonseca, J. Cosmol. Astropart. Phys. 09 (2016) 005.

[23] J. A. Harvey and M. S. Turner, Phys. Rev. D 42, 3344 (1990).

[24] V. Khachatryan et al. (CMS Collaboration), J. High Energy Phys. 02 (2017) 135.

[25] E. Aprile et al. (XENON100 Collaboration), Phys. Rev. Lett. 109, 181301 (2012).

[26] D. S. Akerib et al. (LUX Collaboration), Phys. Rev. Lett. 118, 021303 (2017).

[27] E. Aprile et al. (XENON Collaboration), J. Cosmol. Astropart. Phys. 04 (2016) 027.

[28] G. Angloher et al. (CRESST Collaboration), Eur. Phys. J. C 76, 25 (2016).

[29] CMS Collaboration, CERN Report No. CMS-PAS-HIG-17031, 2018.

[30] G. Aad et al. (ATLAS and CMS Collaborations), J. High Energy Phys. 08 (2016) 045.

[31] M. W. Goodman and E. Witten, Phys. Rev. D 31, 3059 (1985).

[32] J. R. Ellis, K. A. Olive, and C. Savage, Phys. Rev. D 77, 065026 (2008).

[33] Y. Akrami, C. Savage, P. Scott, J. Conrad, and J. Edsjo, J. Cosmol. Astropart. Phys. 04 (2011) 012.

[34] S. Bhattacharya, N. Sahoo, and N. Sahu, Phys. Rev. D 93, 115040 (2016); S. Patra, S. Rao, N. Sahoo, and N. Sahu, Nucl. Phys. B917, 317 (2017); S. Bhattacharya, N. Sahoo, and N. Sahu, Phys. Rev. D 96, 035010 (2017).

[35] J. R. Ellis, A. Ferstl, and K. A. Olive, Phys. Lett. B 481, 304 (2000). 